\title{
The Efficiency of the Hadley Cell Response to Wide Variations in Ocean Heat Transport
}

\author{
M. CAmeron Rencurrel AND Brian E. J. Rose \\ Department of Atmospheric and Environmental Sciences, University at Albany, State University of \\ New York, Albany, New York
}

(Manuscript received 7 May 2019, in final form 28 October 2019)

\begin{abstract}
The Hadley cell (HC) plays a key role in the climate response to variations in ocean heat transport (OHT). Increased OHT is characterized by both a robust slowdown of this overturning circulation, with consequent changes in cloudiness driving the climate response, and a compensating reduction in the atmospheric heat transport (AHT). Here a suite of slab-ocean aquaplanet GCM simulations is used to study the robustness of mechanisms driving changes in HC mass and energy transport across a wide range of idealized spatial patterns of OHT. The HC response is intrinsically related to both the spatial pattern of OHT and the dynamical mechanisms driving the slowdown of the cell. The reduced energy flux of the $\mathrm{HC}$ is associated with reductions in both the mass flux and the gross moist stability (GMS) of the cell in all cases. However, when OHT convergence patterns are confined to the subtropics and equatorward thereof (i.e., subtropical overturning cells), the circulation response is largely momentum-conserving in nature when compared to OHT convergence patterns that extend into the midlatitudes, resulting in a deformation of the anomalous streamfunction following angular momentum contours. The effects of this deformation are quantified through a simple, yet novel approach of splitting the streamfunction anomalies into their "speed" and "shape" components. The tilt of the outer branch of the streamfunction anomaly dampens the direct climate effects of the slowdown of the cell while enhancing the change in GMS, effectively decoupling the change in the energy flux from the slowdown.
\end{abstract}

\section{Introduction}

A key aspect of the climate system is the poleward transport of energy needed to offset the differential heating of Earth's surface by the sun. The majority of this transport occurs in the atmosphere, where the mean meridional circulation (MMC), stationary eddies, and transient eddies all work to transport moist static energy (MSE) poleward overall. In the tropics, atmospheric heat transport (AHT) is primarily accomplished via the Hadley cell (HC), which works to transport warm, moist air equatorward along its lower branch and dry, enhancedpotential-energy air poleward aloft. Because upper-level MSE tends to be greater than at the lower level, the residual transport between the two branches in the tropical free troposphere is poleward.

Both surface and deep ocean currents also work to transport sensible heat out of the tropics. By current

Corresponding author: M. Cameron Rencurrel, crencurrel@ albany.edu estimates, the AHT component dominates poleward of $30^{\circ} \mathrm{N} / \mathrm{S}$, reaching a peak of $5 \mathrm{PW}$ near $43^{\circ}$ while the oceanic heat transport (OHT) is strongest in the deep tropics, with peaks near 2.1 and $1.6 \mathrm{PW}$ at $18^{\circ} \mathrm{N} / \mathrm{S}$ (Trenberth and Caron 2001; Wunsch 2005; Czaja and Marshall 2006).

While OHT in past climates is unconstrained by proxy data (Bice et al. 2000), a number of modeling studies have shown that the tectonic movement of tropical landmasses can give rise to significant changes in OHT through modulations of the tropical oceanic static stability (i.e., depth of the tropical thermocline) (Bice et al. 2000; Hotinski and Toggweiler 2003; Enderton and Marshall 2009; Ferreira et al. 2011). Previous studies of the climatic impact of OHT variations in uncoupled climate models (Winton 2003; Herweijer et al. 2005; Barreiro et al. 2011; Rose and Ferreira 2013; Koll and Abbot 2013) have consistently found that OHT warms the planet while reducing the equator-to-pole temperature gradient, indicating that increased OHT may have been a possible contributor of past warm, equable 
climates such as the late Cretaceous (100-65.5 Ma) and early Eocene (56-34 Ma) (Pearson and Palmer 2000).

Motivated by a number of coupled simulations showing that the meridional profiles of OHT are not fixed and can vary with continental configuration and climatic state (e.g., Hotinski and Toggweiler 2003; Enderton and Marshall 2009; Ferreira et al. 2010, 2011; Rose 2015), Rose and Ferreira (2013) and later Rencurrel and Rose (2018) used an idealized slab-ocean aquaplanet general circulation model (GCM) to analyze the climatic effects of a range of meridional OHT patterns, shown in Fig. 1a. This range is deliberately chosen to more than span the entire plausible range of OHT on Earth, as captured by observations (Trenberth and Caron 2001) and coupled simulations with different continental and climatic configurations (Ferreira et al. 2010; Hotinski and Toggweiler 2003; Rose 2015).

In the slab-ocean framework of Rencurrel and Rose (2018), the convergence of OHT is prescribed as an energy source/sink term (i.e., " $q$-flux") in a shallow ocean mixed layer, shown in Fig. 1b. There is a steady surface heat sink straddling the equator, and an equalbut-opposite heat source in the subtropics or middle to high latitudes (depending on a scale parameter to be described in section 2 below). Figure 1c shows the zonal mean SST response for a 1-PW increase in OHT from 1 to $2 \mathrm{PW}$. Increasing the OHT out of the tropics results in a global mean warming and a reduced equator-to-pole sea surface temperature (SST) gradient in all cases, characterized by nearly invariant equatorial SSTs and extratropical SSTs that depend strongly on the pattern of OHT convergence.

Rencurrel and Rose (2018) found the invariant tropical SSTs to be intrinsically related to the slowdown of the HC (their Fig. 14). Increased OHT out of the tropics (defined as $30^{\circ} \mathrm{S}-30^{\circ} \mathrm{N}$ ), regardless of the spatial pattern, results in decreased convection along the equator in the intertropical convergence zone (ITCZ) tied to the slowdown of the large-scale circulation. Decreased convection results in an increase in downward shortwave (SW) radiation at the surface, offsetting the OHT-driven cooling. Essentially, this mechanism can be described as a competition between cooling by OHT divergence and warming by cloud reductions.

From an energetic perspective, increased OHT was found to associated with a compensating decrease in the AHT, such that the total heat transport (THT) remained relatively constant (Fig. 2a). Rencurrel and Rose (2018) found that in the tropics, atmospheric compensation is predominantly driven by a decrease in the energy flux of the HC.

The brief review above demonstrates that the $\mathrm{HC}$ is a critical component of the climate response to increased OHT, specifically through its role in AHT compensation
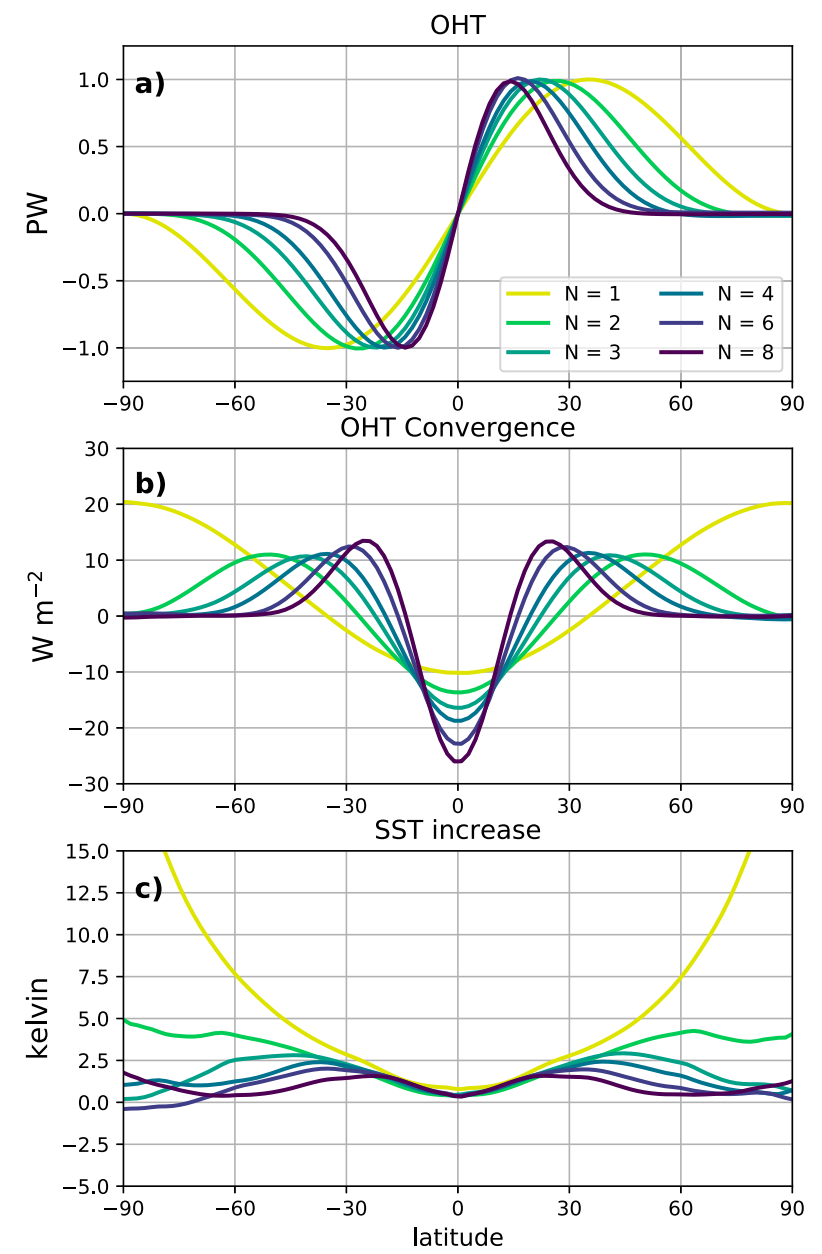

FIG. 1. (a) Idealized ocean heat transport (OHT) profiles from Rencurrel and Rose (2018), shown for different values of the meridional scale parameter $N$ and 1-PW peak transport. (b) Convergence of these profiles $\left(\mathrm{W} \mathrm{m}^{-2}\right.$; the prescribed sea surface heating in our model experiments). (c) SST anomalies for a 1-PW increase in OHT.

and the maintenance of the tropical SSTs. In general, the $\mathrm{HC}$ is one of the most prominent features of the climate system, playing a pivotal role in the shaping of the tropical-to-subtropical climate while working to transport both momentum and energy into the midlatitudes, yet a prognostic, comprehensive theory for the $\mathrm{HC}$ remains elusive (Vallis et al. 2015). Understanding how the $\mathrm{HC}$ responds to a wide variation in OHT will help provide insight into the $\mathrm{HC}$ itself as well as potential past climates.

We calculate the poleward energy flux of the $\mathrm{HC}$ as

$$
F_{\mathrm{HC}}(\phi)=2 \pi a \cos \phi \int_{p_{\mathrm{top}}}^{p_{\mathrm{sfc}}}[\bar{m}][\bar{v}]_{\mathrm{adj}} \frac{d p}{g},
$$

with $m$ the MSE, $v_{\text {adj }}$ the meridional wind adjusted for mass imbalance over the entire column following 

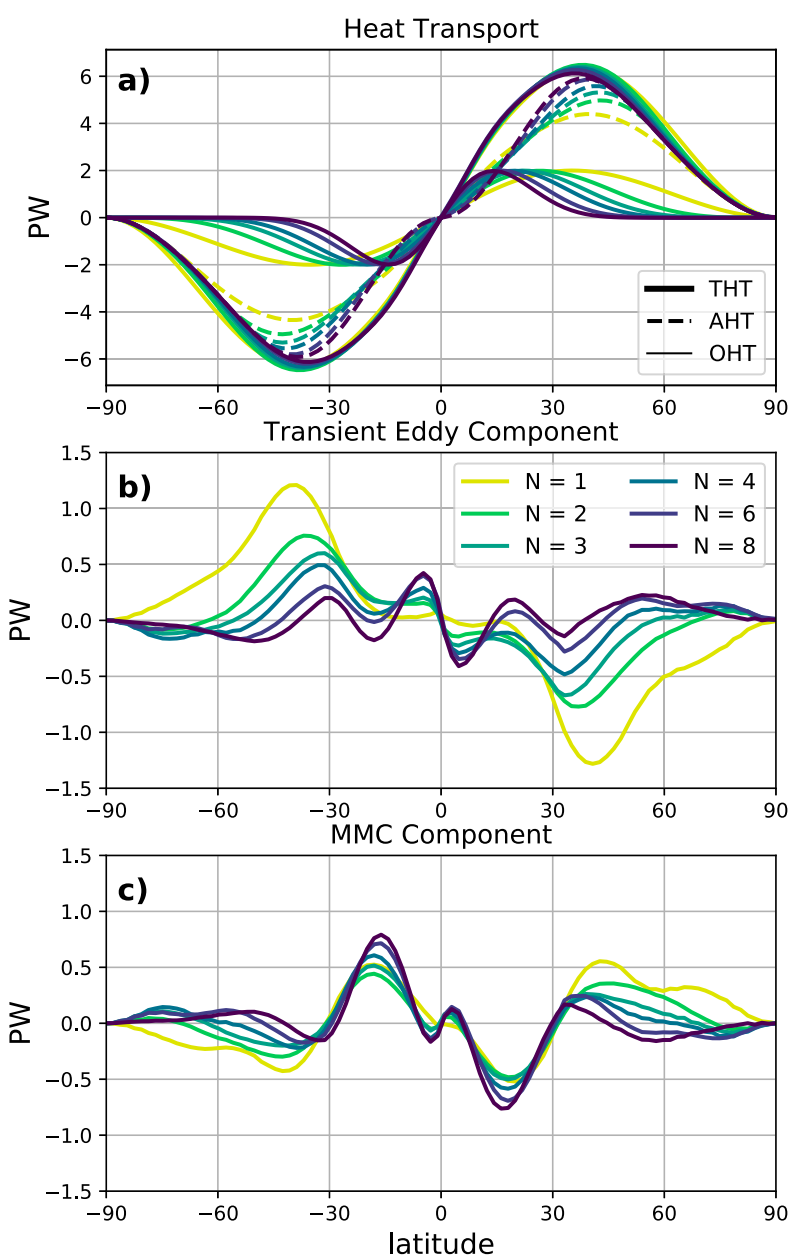

FIG. 2. (a) Total (THT), atmospheric (AHT), and ocean heat transport (OHT) components for all $N$ at $2 \mathrm{PW}$. (b) Transient eddy heat transport anomaly for a 1-PW increase in OHT. (c) As in (b), but for the mean meridional circulation (MMC). Note that the $y$-axis scale for the anomalies is smaller than for the initial state.

Hill et al. (2015), $p_{\text {top }}$ and $p_{\text {sfc }}$ the pressure at the tropopause and surface, and the square brackets and overbars representing the zonal mean and the time mean, respectively. It is important to note that this energy flux is dependent on not just the circulation of the cell $\bar{v}$ but on its relationship with the energy field $\bar{m}$, which itself is dependent on the spatial pattern of OHT. The key question for this paper is, What role does the $\mathrm{HC}$ play in the atmospheric compensation, and how do the mechanisms depend on the spatial pattern of OHT?

From a dynamical perspective, there are two separate, incomplete theories that can be used to describe changes in the strength of the overturning circulation. In the angular momentum-conserving limit, Held and Hou (1980) showed that variations in a thermally direct $\mathrm{HC}$ are primarily driven by changes in the tropopause height, the fractional temperature difference between the equator and poles, and the atmospheric stability. On the other hand, Walker and Schneider (2006) showed that variations in the $\mathrm{HC}$ are largely driven by eddy transport of momentum out of the tropics, tied to the strength of midlatitude baroclinicity. Aspects of both of these theories have been proven to be correct in the actual climate system, yet neither fully capture the driving mechanisms of the HC (Schneider 2006). Given the spatial dependence of the SST response to OHT perturbations (Fig. 1c), it is possible that the slowdown mechanisms span both possible theories. In line with the above question, then, are the following: What are the dynamical mechanisms driving the slowdown of the HC, how are they dependent on the spatial pattern of the prescribed forcing, and what roles do they play in setting the HC energy flux?

Here, we seek to answer these questions by analyzing the $\mathrm{HC}$ response to increases in OHT using the same set of aquaplanet simulations from Rencurrel and Rose (2018). We find not only that the HC's role in atmospheric compensation is dependent on the spatial pattern of the prescribed forcing, but also that it is inherently dependent on the dynamical mechanisms driving the slowdown of the cell as well. Equator-to-subtropical-scale OHT (large $N$ ) results in a more thermally direct, momentum-conserving slowdown-in the sense that anomalous streamlines are largely parallel to angular momentum contours in the free troposphere-than does a larger-scale OHT pattern. The momentum-conserving nature of this slowdown results in a change in shape in the anomalous streamfunction, tilting to follow angular momentum contours. The effects of this deformation are quantified through a simple, yet novel approach of splitting the streamfunction anomalies into their "speed" and "shape" components, defined in section 3. We find that the change in shape of the streamfunction anomaly reduces the relative strength of the near-surface MSE gradient that is advected by the circulation [ $v$ in (1)], effectively decoupling the HC energy flux decrease from the slowdown of the circulation. As we will show, the mechanisms by which the HC transports energy out of the tropics are dependent on its momentum transport.

The rest of this paper is organized as follows. Section 2 describes the GCM used for this analysis along with the prescribed OHT patterns. The HC's role in atmospheric compensation is discussed in section 3. Section 4 takes a closer look at $\mathrm{HC}$ energy transport efficiency, which is related to the dynamical mechanisms driving the $\mathrm{HC}$ slowdown in section 5. Discussions and conclusions follow in section 6 .

\section{Experimental setup}

We use an atmospheric GCM coupled to an aquaplanet mixed layer ocean with a steady, zonally symmetric 
prescribed heat source/sink term (the $q$-flux) representing regions of convergence and divergence of OHT, as shown in Fig. 1b. The OHT patterns are identical to those analyzed by Rencurrel and Rose (2018), following the simple formulation from Rose and Ferreira (2013):

$$
\mathrm{OHT}=\Psi \sin (\phi) \cos (\phi)^{2 N}=\Psi x\left(1-x^{2}\right)^{N},
$$

where $x=\sin (\phi), \phi$ is latitude, $N$ is a positive integer that sets the meridional extent of OHT, and $\Psi$ is a constant (W) that sets the amplitude of the prescribed pattern.

The value of $N$ is set to range from 1 to 8 , as shown in Fig. 1a, simulating OHT patterns ranging from subtropical to polar in nature. Rencurrel and Rose (2018) considered a wide range of amplitudes of OHT (from 0 to $4 \mathrm{PW}$ ), chosen to more than span the plausible range of realistic patterns. In the present work we focus more narrowly on the climate response to an OHT increase from 1 to $2 \mathrm{PW}$ to avoid issues with unrealistically high subtropical OHT magnitudes of 3 to 4 PW (Rencurrel and Rose 2018). This range nearly brackets the 1.6-2.1-PW values for the two hemispheres in modern observations (Trenberth and Caron 2001) while effectively capturing potential past/future amplitude variations (Enderton and Marshall 2009; Ferreira et al. 2010; Rose 2015). Most of our figures, beginning with Fig. 1c, show anomalies for a 1-PW increase in OHT (peak transport increasing from 1 to $2 \mathrm{PW}$ ).

The GCM used is the Community Atmospheric Model, version 4 (CAM4), the atmospheric component of the modular Community Earth System Model (CESM). We use CAM4 in its standard configuration regarding horizontal resolution $\left(2.0^{\circ} \times 2.5^{\circ}\right)$, vertical resolution $(26$ levels), and physical parameterizations [including the Zhang-McFarlane deep convection scheme as modified by Neale et al. (2013)]. The model has fully interactive clouds and water vapor, and thus incorporates many of the fundamental radiative feedback processes absent in past idealized gray radiation aquaplanet studies. Reference greenhouse gases are 348 ppmv $\mathrm{CO}_{2}, 1650$ ppbv $\mathrm{CH}_{4}$, and 306 ppbv $\mathrm{N}_{2} \mathrm{O}$ with all other greenhouse gases set to zero. Ozone is prescribed as in Blackburn and Hoskins (2013). The surface albedo is fixed at 0.1. Sea ice is omitted and SST is permitted to drop below freezing. These specifications follow previous aquaplanet intercomparison protocols (Lee et al. 2008; Rose et al. 2014; Voigt et al. 2016). The model is set to a perpetual equinox state with a $0^{\circ}$ obliquity and a $10-\mathrm{m}$ mixed layer depth, identical to the multimodel comparison analyzed in Rose et al. (2014) and Rose and Rencurrel (2016). Simulations are run for 30 years with an averaging period over the last 20 years.
We choose to exclude sea ice from these simulations because previous studies have shown that the coupling between sea ice and OHT convergence patterns is extraordinarily strong in aquaplanet simulations (e.g., Rose 2015) and we wish to study the effects of OHT on atmospheric radiative and dynamical processes without the confounding effect of very large changes in sea ice extent. This exclusion is consistent with many previous aquaplanet studies (Lee et al. 2008; Rose et al. 2014; Voigt et al. 2016; Rose and Rencurrel 2016).

\section{Atmospheric compensation}

Here, we look at the compensating changes of AHT to an increase in OHT as well as the HC's role in driving the response. Figure 2 a shows a fairly robust THT pattern across all runs, largely insensitive to variations in OHT. THT is calculated energetically from the TOA radiative fluxes:

$$
F_{\mathrm{THT}}(\phi)=2 \pi a^{2} \int_{-\pi / 2}^{\phi}\left[\bar{Q}_{\mathrm{TOA}}\right] \cos \phi d \phi,
$$

while AHT is calculated from the residual between the TOA and surface heat fluxes:

$$
F_{\mathrm{AHT}}(\phi)=2 \pi a^{2} \int_{-\pi / 2}^{\phi}\left(\left[Q_{\mathrm{TOA}}\right]-\left[Q_{\mathrm{sfc}}\right]\right) \cos \phi d \phi,
$$

with $Q_{\mathrm{TOA}}$ and $Q_{\mathrm{sfc}}$ being the downward energy flux at the TOA and surface, and $a$ the planetary radius.

Compensating changes in meridional energy transport have long been shown to be a fundamental aspect of the climate system (Stone 1978; Bjerknes 1964). In the steady state, THT is uniquely determined by the meridional divergence of the net top-of-atmosphere (TOA) radiative forcing, primarily set by astronomical parameters and albedo, and is largely insensitive to variations in either OHT or AHT (Armour et al. 2019). Because we impose surface $q$-flux perturbations with zero global mean, compensation must be a fundamental aspect of the equilibrium response. However, this compensation is not perfect. Increasing OHT in these model runs is characterized by a slight increase in the THT and an undercompensation in the AHT response in all cases. This can be attributed to an increase in the meridional TOA radiative gradient driven primarily by cloud feedbacks (Rencurrel and Rose 2018; Liu et al. 2016).

Assuming transport of kinetic energy to be negligible, we use (1) to calculate the mean meridional circulation (MMC) component of the transport, comprising the $\mathrm{HC}$ in the tropics and the Ferrel cell in the midlatitudes (Trenberth and Stepaniak 2003; Hill et al. 2015). Because stationary eddies are negligible on a zonally symmetric 

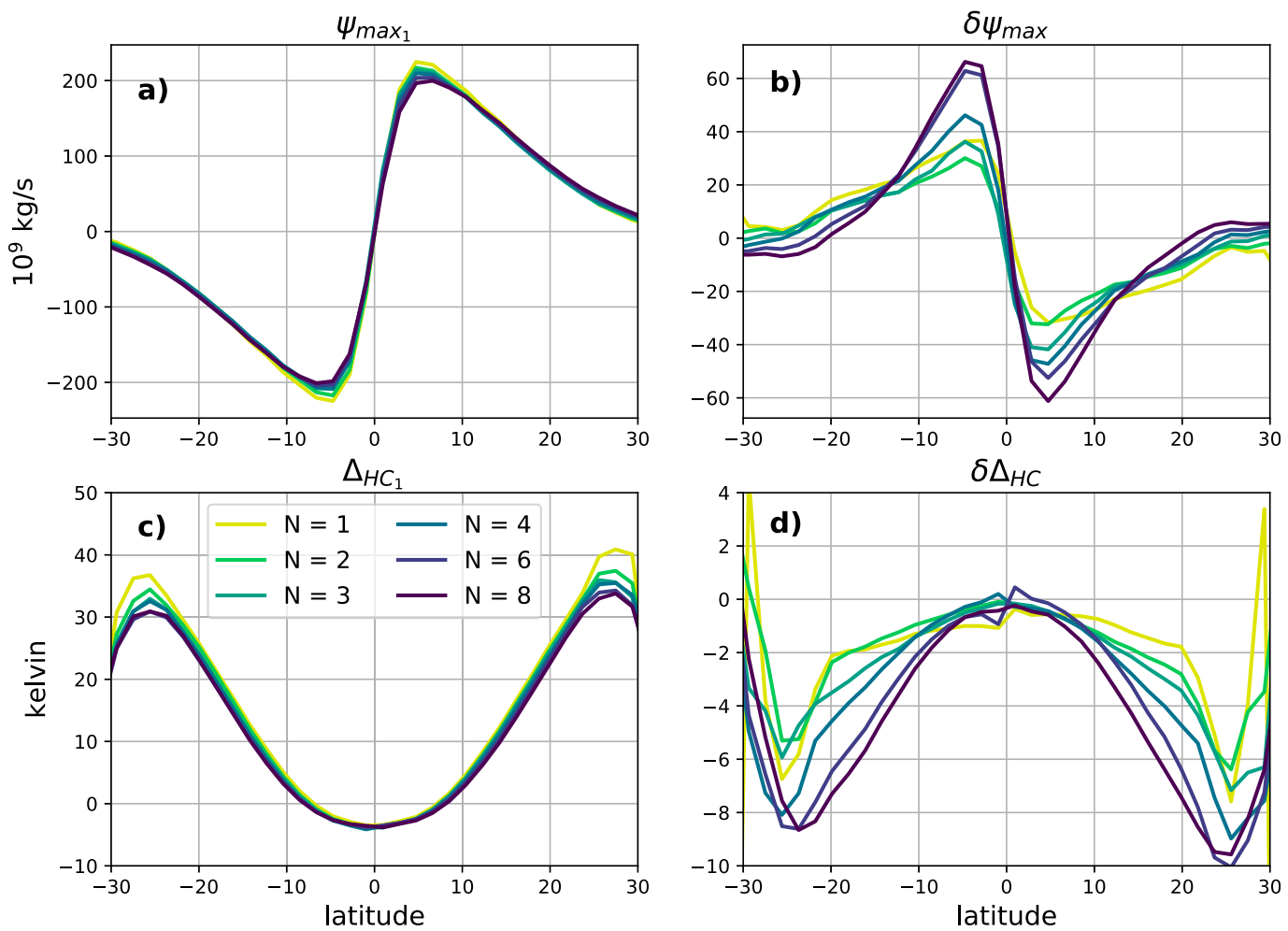

FIG. 3. (a) Initial HC mass flux for prescribed 1-PW OHT. (b) Anomalous poleward Hadley cell mass flux for a 1-PW increase in OHT. (c) Initial GMS for prescribed 1-PW OHT. (d) Anomalous gross moist stability (GMS). GMS has been divided by $c_{p}$ to present results in units of kelvin. Initial states are an order of magnitude greater than the respective anomalies. The latitude range is limited to $\pm 30^{\circ}$ here and in the remaining of the figures.

aquaplanet, the transient eddy component is simply taken to be the residual between (4) and (1). This partitioning for the anomalous AHT is plotted in Figs. $2 b$ and 2c. The AHT adjustment is largely a local response to OHT variations, in the sense that changes in AHT tend to be equal and opposite to changes in OHT and thus occur farther poleward for small $N$ (midlatitude forcing). The primary mechanism for this compensation shifts from MMC for large $N$ to transient eddies for small $N$. This is perhaps not surprising, as the $\mathrm{HC}$ and transient eddies are largely tropical and midlatitude phenomena respectively. However, the MMC component decreases robustly by $0.5-0.7 \mathrm{PW}$ in the subtropics for all $N$.

We relate this compensating energy flux to the slowdown of the mass flux by rewriting (1) as

$$
F_{\mathrm{HC}}(\phi)=\psi_{\max }(\phi) \Delta_{\mathrm{HC}}(\phi),
$$

with $\psi_{\max }(\phi)$ as the lower-level mass flux of the circulation, calculated as the signed maximum of the Eulerian meridional mass streamfunction $\psi$, from

$$
\psi(\phi, p)=2 \pi a \cos \phi \int_{0}^{p}[\bar{v}]_{\mathrm{adj}} \frac{d p}{g},
$$

and $\Delta_{\mathrm{HC}}(\phi)$ as the efficiency of the HC energy transport per unit mass transport. In (5) $\psi_{\max }$ represents the poleward mass flux of the cell, directly tied to the strength of the overturning circulation. The initial mass flux for a prescribed OHT of $1 \mathrm{PW}$ and the change in the mass flux for an increase in OHT to $2 \mathrm{PW}$ are shown in Figs. $3 \mathrm{a}$ and $3 \mathrm{~b}$ respectively. Increasing OHT results in a $10 \%-30 \%$ reduction in the maximum strength of the overturning circulation. Subtropical forcing (large $N$ ) is characterized by a greater slowdown than its midlatitude counterparts, generally increasing with $N(N=1$ being the exception, discussed later in section 5).

The term $\Delta_{\mathrm{HC}}$ is also known as the gross moist stability (GMS) of the HC. GMS governs how well the HC is able to transport energy out of the tropics, also calculated as the meridional flow-weighted difference between the upper-level and lower-level MSE (Neelin and Held 1987). The initial GMS is shown in Fig. 3c. In all cases, we find GMS to be slightly negative in the deep tropics, increasing poleward toward the $\mathrm{HC}$ edge. While not realistic, negative GMS in the deep tropics is a common occurrence in aquaplanet simulations (Frierson 2007).

Changes in GMS can influence the change in the HC circulation needed to reach equilibrium in response to a 

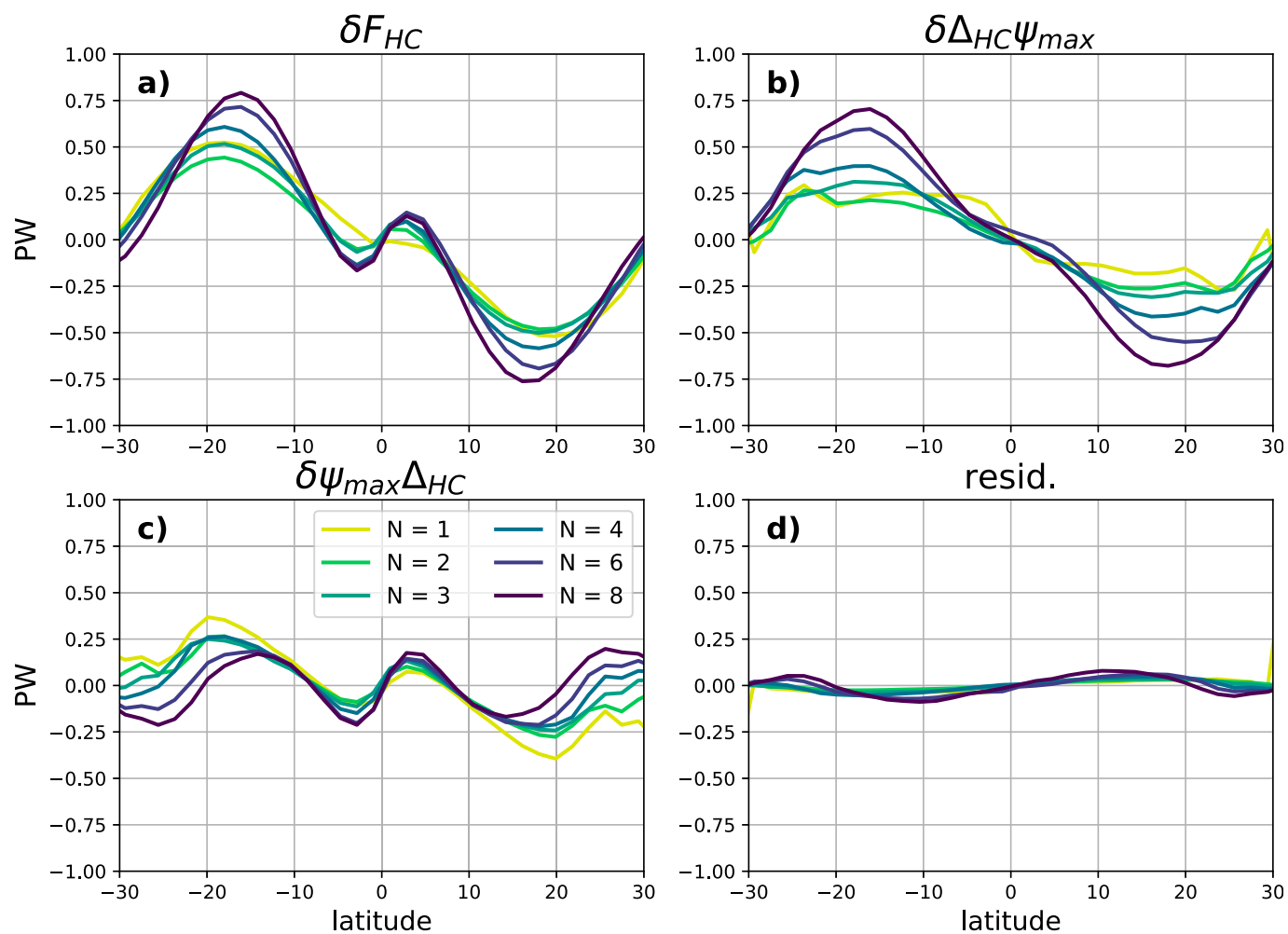

FIG. 4. Linear breakdown of anomalous Hadley cell energy flux components. (a) Total HC energy flux anomalies, (b) thermodynamic efficiency-driven energy flux anomalies, (c) dynamic circulation-driven energy flux anomalies, and (d) nonlinear residual.

climate forcing. In the case of a positive correlation between the mass flux and the GMS, the change in GMS will partially compensate for the induced energy imbalance, resulting in a weaker mass circulation response (Hill et al. 2015; Ma et al. 2018). The change in GMS calculated from (5) is shown in Fig. 3d. Increasing OHT results in a $10 \%-40 \%$ decrease in GMS at $15^{\circ} \mathrm{N}$. Similar to the mass flux of the cell, large $N$ is characterized by a greater decrease in the efficiency of the energy transport.

Both the reduction in the GMS and the slowdown of the overturning circulation are working to reduce the HC poleward energy flux. To determine the relative importance of these two mechanisms, we linearly expand (5) such that

$$
\begin{aligned}
\delta F_{\mathrm{HC}}(\phi)= & \psi_{\max }(\phi) \delta \Delta_{\mathrm{HC}}(\phi)+\delta \psi_{\max }(\phi) \Delta_{\mathrm{HC}}(\phi) \\
& + \text { residual. }
\end{aligned}
$$

The first right-hand-side term represents the decreased energy flux associated with the decreased transport efficiency. As expected, a reduction in GMS results in a decrease in the $\mathrm{HC}$ poleward energy transport, shown in Fig. 4b. And in the cases of subtropical OHT forcing (large $N$ ), a larger decrease in the transport efficiency results in a greater reduction in the energy flux.
Curiously, this simplicity does not extend to the second term in (7), the change in the energy flux associated with variations in the overturning circulation, shown in Fig. 4c. Away from the equator, the slowdown of the $\mathrm{HC}$ is working to reduce the energy flux of the cell, but a greater slowdown does not result in a greater energy flux decrease. On the contrary, midlatitude and polar OHT forcing patterns (small $N$ ) appear to have the greatest effect, despite the weaker circulation response. The nonlinear interactions between the two components are negligible (Fig. 4d).

Making the qualitatively sound assumption that the majority of GMS is primarily set by the near-surface MSE gradient (Held 2001; Hill et al. 2015; Merlis et al. 2013), we can study this breakdown by analyzing the relationship between the streamfunction and the MSE field, shown in the left two panels of Fig. 5 for two illustrative example cases: midlatitude $(N=2)$ and subtropical $(N=6)$ OHT forcing. (The full breakdown for all $N$ is appended in Fig. A1.)

The leftmost panels show the relationship between the anomalous MSE field and the reference streamfunction (taken from the 1-PW case for each $N$ ), representative of the efficiency-driven decrease in the energy flux. Increased OHT works to enhance near-surface MSE 

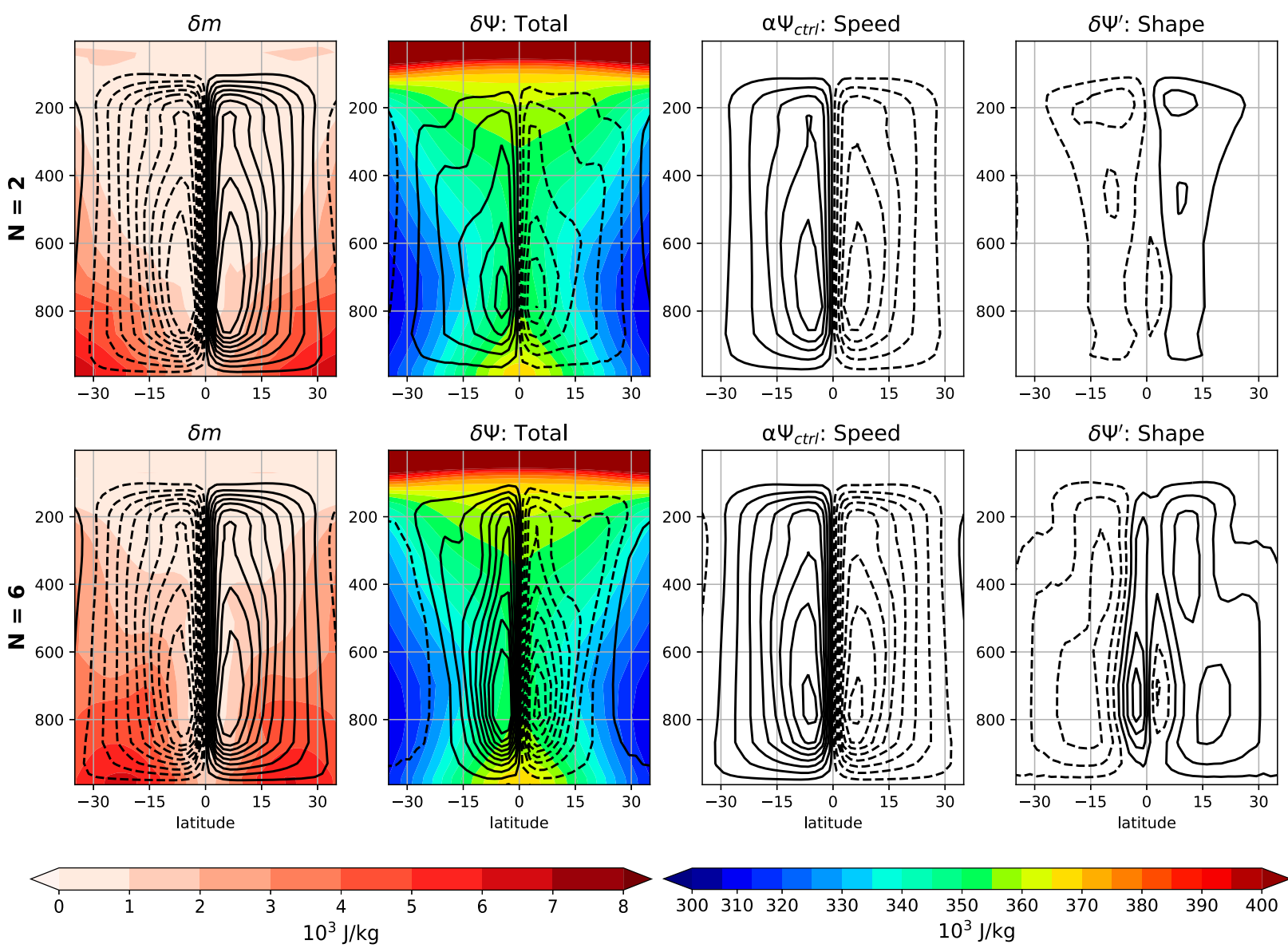

FIG. 5. (left) Anomalous moist static energy (MSE) plotted with reference streamfunction (contour interval: $20 \times 10^{9} \mathrm{~kg} \mathrm{~s}^{-1}$ ), $($ center left) anomalous streamfunction (contour interval: $6 \times 10^{9} \mathrm{~kg} \mathrm{~s}^{-1}$ ) plotted with reference MSE field, and (center right) speed and (right) shape components of the streamfunction anomaly for a 1-PW OHT increase for (top) $N=2$ and (bottom) $N=6$.

primarily in the regions of OHT convergence, reducing the GMS (i.e., transport efficiency) through the increase in the equatorward energy flux of the lower branch of the HC. Increased equatorward transport works to offset the poleward energy transport of the upper branch, reducing how much energy the $\mathrm{HC}$ is able to transport poleward per unit mass flux. This effect is greater in the case of subtropical forcing $(N=6)$, where the maximum MSE anomaly is directly interacting with the circulation.

The center-left panels represent the dynamically driven energy flux relationship between the anomalous streamfunction and the reference MSE field. Subtropical OHT forcing $(N=6)$ results in a greater decrease in $\mathrm{HC}$ mass flux when compared to its midlatitude counterpart $(N=2)$. This slowdown works to decrease the energy flux of the cell. However, this enhanced slowdown is also characterized by a reduction in the meridional extent of the surface branch of the streamfunction anomaly, indicating a decrease in magnitude of the MSE gradient with which the anomalous flow is interacting.
This results in a decrease in amplitude of the dynamically driven $\mathrm{HC}$ heat transport anomaly, partially offsetting the anomalous flow associated with the greater slowdown.

To quantify this effect, we conduct a simple, yet novel breakdown of the streamfunction anomaly in which we separate it into its "speed" $\psi_{\text {sp }}$ and "shape" $\psi_{\text {sh }}$ components, with

$$
\begin{aligned}
\delta \psi_{\mathrm{sp}} & =\alpha \psi_{\mathrm{ref}}, \\
\alpha & =\frac{\max (\delta \psi)}{\max \left(\psi_{\mathrm{ref}}\right)},
\end{aligned}
$$

and

$$
\delta \psi_{\mathrm{sh}}=\delta \psi-\delta \psi_{\mathrm{sp}}
$$

where $\psi_{\text {ref }}$ is the reference streamfunction for each $N$. The term $\delta \psi_{\text {sp }}$ represents the change in strength of the streamfunction assuming a fixed circulation pattern, calculated as the scaling of the reference streamfunction 

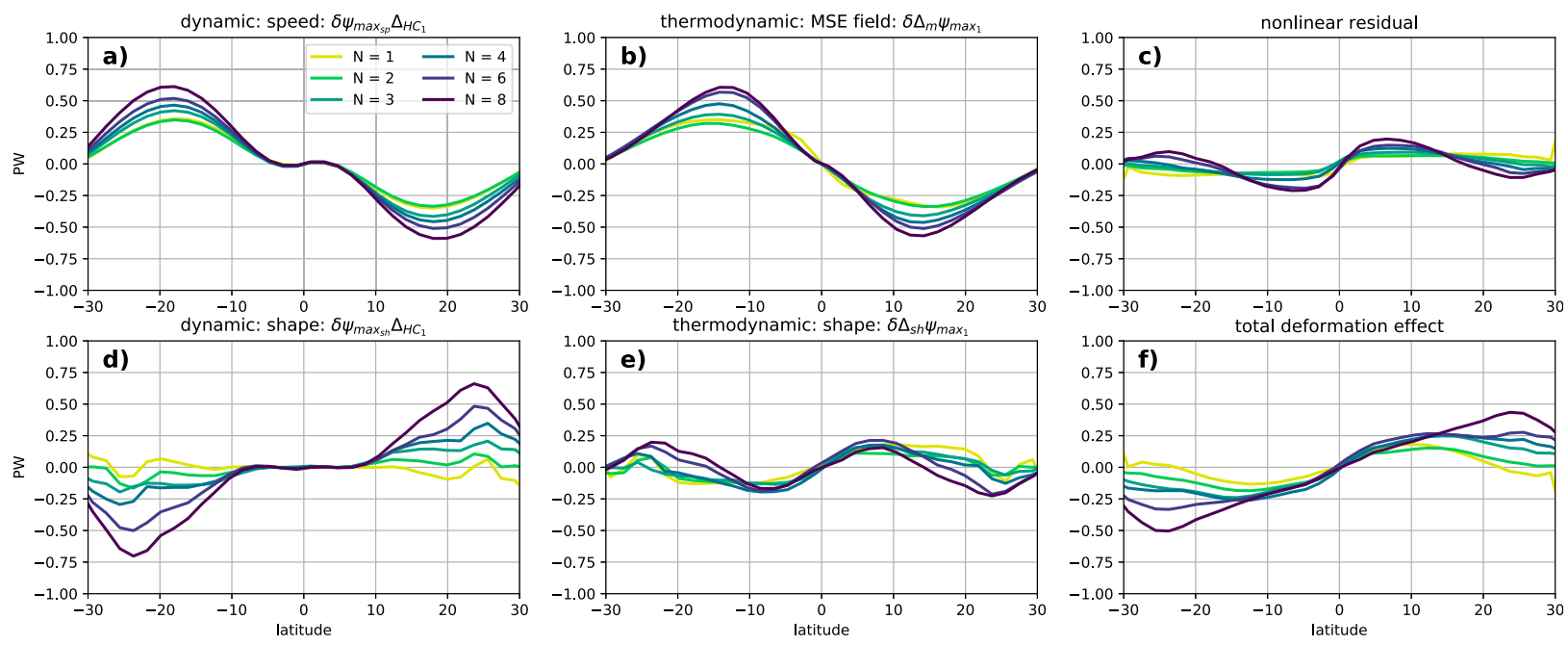

FIG. 6. Full linear breakdown of anomalous Hadley cell energy flux components. Transport changes driven by (a) the direct effect of the physical slowdown of the HC, (b) the change in GMS associated with the anomalous MSE field, (c) nonlinear mechanisms, (d) the direct effect of the deformation of the HC anomaly, (e) the change in GMS associated with the deformation of the HC anomaly, and (f) total effect of the HC deformation anomaly [sum of (d) and (e)].

by the maximum fractional change $\alpha ; \delta \psi_{\mathrm{sp}}$ represents the change in the shape of the streamfunction, calculated as a residual of the total anomalous streamfunction $\delta \psi$ and the speed component $\delta \psi_{\text {sp. }}$. The "speed" and "shape" components of the streamfunction anomaly are shown in the center-right and far right panels of Fig. 5 respectively. Subtropical OHT forcing $(N=6)$ results in both a greater slowdown and greater deformation compared to its midlatitude counterpart $(N=2)$.

We can directly calculate the effects of the HC slowdown and deformation on the anomalous energy transport by expanding the dynamically driven term in (7):

$$
\begin{aligned}
\delta \psi_{\max }(\phi) \Delta_{\mathrm{HC}}(\phi)= & \delta\left(\psi_{\mathrm{sp}}\right)_{\max }(\phi) \Delta_{\mathrm{HC}}(\phi) \\
& +\delta\left(\psi_{\mathrm{sh}}\right)_{\max }(\phi) \Delta_{\mathrm{HC}}(\phi),
\end{aligned}
$$

with $\delta\left(\psi_{\mathrm{sp}}\right)_{\max }(\phi)$ and $\delta\left(\psi_{\mathrm{sh}}\right)_{\max }(\phi)$ representing the anomalous mass flux associated with the speed and shape components of the $\mathrm{HC}$ anomaly respectively. To effectively capture the mass flux anomalies, we calculate the anomalous mass flux components as the difference in the mass fluxes associated with the initial streamfunction and the streamfunction assuming just the change in the speed/shape of the cell (e.g., $\left.\psi_{\mathrm{ref}}+\psi_{\mathrm{sh}}\right)$. The results of this breakdown are shown in Figs. 6a and 6d. The enhanced slowdown associated with subtropical OHT forcing (large $N$ ) does result in a greater decrease in the $\mathrm{HC}$ energy flux when compared to the extratropical forcing patterns (small $N$ ), but this difference is clearly offset by the associated deformation of the streamfunction anomaly, resulting in the disconnect between the decrease in the $\mathrm{HC}$ energy flux and its dynamically driven component, as shown in Fig. 4c.

As the OHT anomalies shift toward the subtropics ( $N$ increases), there is a clear reduction in the HC energy flux associated with the increased slowdown of the overturning circulation. However, this reduction is offset by the change in the shape of the $\mathrm{HC}$, resulting in the disconnect between the overall decreased $\mathrm{HC}$ energy flux and its dynamically driven component.

\section{Transport efficiency}

We have demonstrated that the deformation of the streamfunction anomaly associated with subtropical OHT forcing patterns (large $N$ ) works to disconnect the physical slowdown of the $\mathrm{HC}$ and its energy flux reduction. Because of this, the majority of the HC energy flux decrease is tied to the decrease in GMS, or the reduction in the efficiency of the heat transport, as shown in Fig. 4b. It is worth asking then, What role does the deformation of the streamfunction anomaly have on the GMS response?

As mentioned previously, changes in GMS can be qualitatively understood through changes in the MSE field. More specifically, Held (2001) assumes that GMS can be captured by the difference in the near-surface MSE locally and at the ITCZ:

$$
\Delta_{\mathrm{HC}_{\mathrm{Held}}}(\phi) \approx m_{\mathrm{ITCZ}}-m(\phi),
$$



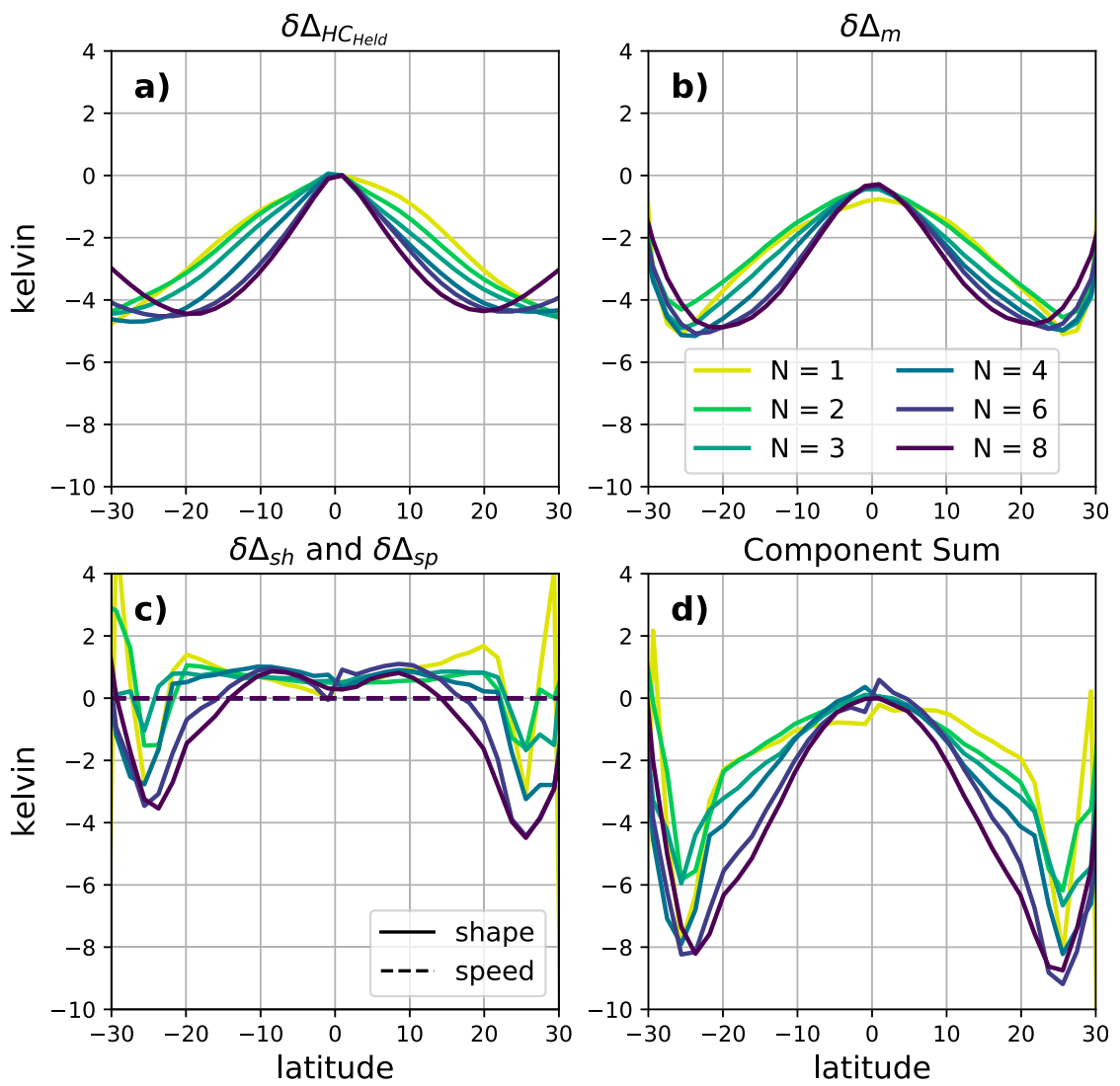

FIG. 7. (a) Approximate gross moist stability anomaly calculated from (11) (Held 2001). Component breakdown of the anomalous Hadley cell energy transport efficiency: (b) decreased efficiency associated with the change in the moist static energy field, (c) decreased efficiency associated with both the slowdown and the deformation of the Hadley cell anomaly, and (d) the sum of the three components [in (b) and (c)]. GMS has been divided by $c_{p}$ to present results in units of kelvin.

based on 1) vertical homogenization of MSE within the ITCZ by convection and horizontal homogenization by gravity waves in the upper troposphere, and 2) equal mass flux weighting between the upper and lower branches.

Under this assumption, the decrease in GMS can be attributed to the increase in the near-surface MSE in regions of OHT convergence away from the ITCZ. However, it is clear that in the case of subtropical forcing (high $N$ ), the mass flux weighting between the upper and lower HC branches no longer cancels and (11) is not a valid assumption. We illustrate this misfit by calculating the approximated GMS from (11), shown in Fig. 7a. In the cases of subtropical forcing, the decrease in the nearsurface MSE gradient only accounts for approximately $50 \%$ of the total change in GMS.

To quantify the effect of the cell deformation on the GMS anomaly, we first must expand (5) in terms of both the MSE and the streamfunction. From (6), we can show that

$$
\bar{v}=\frac{g}{2 \pi a \cos \phi} \frac{\partial \bar{\psi}}{\partial p}
$$

with $\bar{v}$ and $\bar{\psi}$ representing the time and zonal-mean meridional wind velocity and streamfunction respectively. Plugging (12) into (1) allows us to calculate the HC energy flux in terms of the streamfunction as well as the MSE field, which we can then divide by the mass flux of $\bar{\psi}$ to get the GMS:

$$
\Delta_{\mathrm{HC}}=2 \pi a \cos \phi \int_{p_{\mathrm{top}}}^{p_{\mathrm{sc}}}[\bar{m}]\left[\frac{g}{2 \pi a \cos \phi} \frac{\partial \bar{\psi}}{\partial p}\right]_{\mathrm{adj}} \frac{d p}{g} \psi_{\mathrm{max}}^{-1} .
$$

Assuming the linearity illustrated in (7) is robust, we can calculate the direct effect of the change in the MSE and streamfunction individually by holding the opposing term constant. We can also further break down the streamfunction-driven change in the GMS by isolating the changes in the speed and shape component of the cell, as in the previous section. 
Holding the streamfunction fixed,

$\delta \Delta_{m}=2 \pi a \cos \phi \int_{p_{\mathrm{top}}}^{p_{\mathrm{sfc}}}\left[\bar{m}_{2}\right]\left[\frac{g}{2 \pi a \cos \phi} \frac{\partial \overline{\psi_{1}}}{\partial p}\right]_{\mathrm{adj}} \frac{d p}{g} \psi_{\text {max }_{1}}^{-1}-\Delta_{\mathrm{HC}_{1}}$

is the change in the GMS associated with the increased MSE, with the subsets 1 and 2 representing the amplitude (PW) of the prescribed OHT pattern in each state. By holding the MSE fixed,

$$
\begin{aligned}
\delta \Delta_{\mathrm{sh}}= & 2 \pi a \cos \phi \int_{p_{\mathrm{top}}}^{p_{\mathrm{sfc}}}\left[\bar{m}_{1}\right]\left[\frac{g}{2 \pi a \cos \phi} \frac{\partial \overline{\mathrm{sh}_{2}}}{\partial p}\right]_{\mathrm{adj}} \frac{d p}{g} \psi_{\text {max }_{\mathrm{sh} 2}^{-1}} \\
& -\Delta_{\mathrm{HC}_{1}}
\end{aligned}
$$

and

$$
\begin{aligned}
\delta \Delta_{\mathrm{sp}}= & 2 \pi a \cos \phi \int_{p_{\mathrm{top}}}^{p_{\mathrm{sfc}}}\left[\bar{m}_{1}\right]\left[\frac{g}{2 \pi a \cos \phi} \frac{\partial \overline{\mathrm{sp}}}{\partial p}\right]_{\mathrm{adj}} \frac{d p}{g} \psi_{\mathrm{max}_{\mathrm{sp} 2}^{-1}} \\
& -\Delta_{\mathrm{HC}_{1}}
\end{aligned}
$$

are the change in the GMS driven by the change in the shape and speed of the cell respectively. We find that

$$
\delta \Delta_{\mathrm{HC}} \approx \delta \Delta_{m}+\delta \Delta_{\mathrm{sh}}+\delta \Delta_{\mathrm{sp}} .
$$

The results of this breakdown are shown in Figs. 7b-d. As expected, the change in the MSE field only accounts for approximately $50 \%$ of the decreased GMS in the subtropics, similar to the approximated GMS calculated in Fig. 7a. The rest of the change can be attributed to the change in the shape of the streamfunction anomaly. As expected, the change in the speed component is zero. All else being fixed, increasing or decreasing the rate of the overturning circulation has no effect on how efficient the cell is at transporting energy poleward.

Just as with the dynamically driven terms in the previous section, we can directly calculate the effects of this GMS enhancement on the anomalous energy transport by expanding the efficiency-driven term in (7):

$$
\psi_{\max }(\phi) \delta \Delta_{\mathrm{HC}}(\phi) \approx \psi_{\max }(\phi) \delta \Delta_{m}(\phi)+\psi_{\max }(\phi) \delta \Delta_{\mathrm{sh}}(\phi),
$$

shown in Figs. 6b and 6e. As expected, the change in the MSE field has the greatest influence on the energy transport through the decreased transport efficiency. Subtropical OHT convergence works to warm the lower troposphere, enhancing the MSE transported equatorward by the lower branch of the HC. This effect is further enhanced in the subtropics by the deformation of the cell, where the change in shape of the streamfunction works to reduce the magnitude of the MSE gradient with which the anomalous surface flow is interacting.

The reduction in the meridional extent of the surface branch works both to offset the direct effects of the slowdown of the cell and to enhance the decreased GMS, making the compensating change in the energy flux largely efficiency-driven in nature. However, in analyzing the sum of these two components (shown in Fig. 6f), we find that the change in shape of the $\mathrm{HC}$ results in an overall net decrease in the energy flux of the cell. The enhanced GMS anomaly does not fully offset the direct dynamical effects of this feature.

\section{Deformation mechanisms}

We have shown that the deformation of the streamfunction plays a crucial role in setting the HC energy flux response to an increase in OHT, effectively decoupling the energy flux decrease from the mass flux decrease while enhancing the change in GMS. The next logical questions are then, What is driving the deformation of the $\mathrm{HC}$, and how is it dependent on the spatial pattern of the applied OHT forcing?

The top panels of Fig. 8 show the anomalous streamfunction for the cases of midlatitude $(N=2)$ and subtropical $(N=6)$ forcing plotted against the angular momentum contours of the reference states. The anomalous cell deformation in the subtropical case is actually a tilt in the outer branch of the $\mathrm{HC}$ along the angular momentum contours, indicative of an anomalous flow with very limited momentum advection.

Such a tilt in the outer branch of the streamfunction has been shown to be a robust feature in a number of momentum-conserving, two-dimensional axisymmetrical models of the HC (Held and Hou 1980; Satoh 1994; Fang and Tung 1996; Singh and Kuang 2016; Singh et al. 2017). In the inviscid limit, angular momentum within the $\mathrm{HC}$ is homogenized to its value within the ascent region. As a result, the $\mathrm{HC}$ can only be in contact with surface where the angular momentum of the circulation matches that of the lower boundary near the equator, giving us the deformed pattern we find in these cases. In the case of midlatitude forcing, shown in the top-left panel of Fig. 8, flow across the angular momentum contours is indicative of a non-momentum-conserving streamfunction anomaly. (The relationship between the streamfunction anomalies and the angular momentum contours for all $N$ is appended in Fig. A2.)

This drastic change in angular momentum advection with $N$ is indicative of a shift in the dynamical 

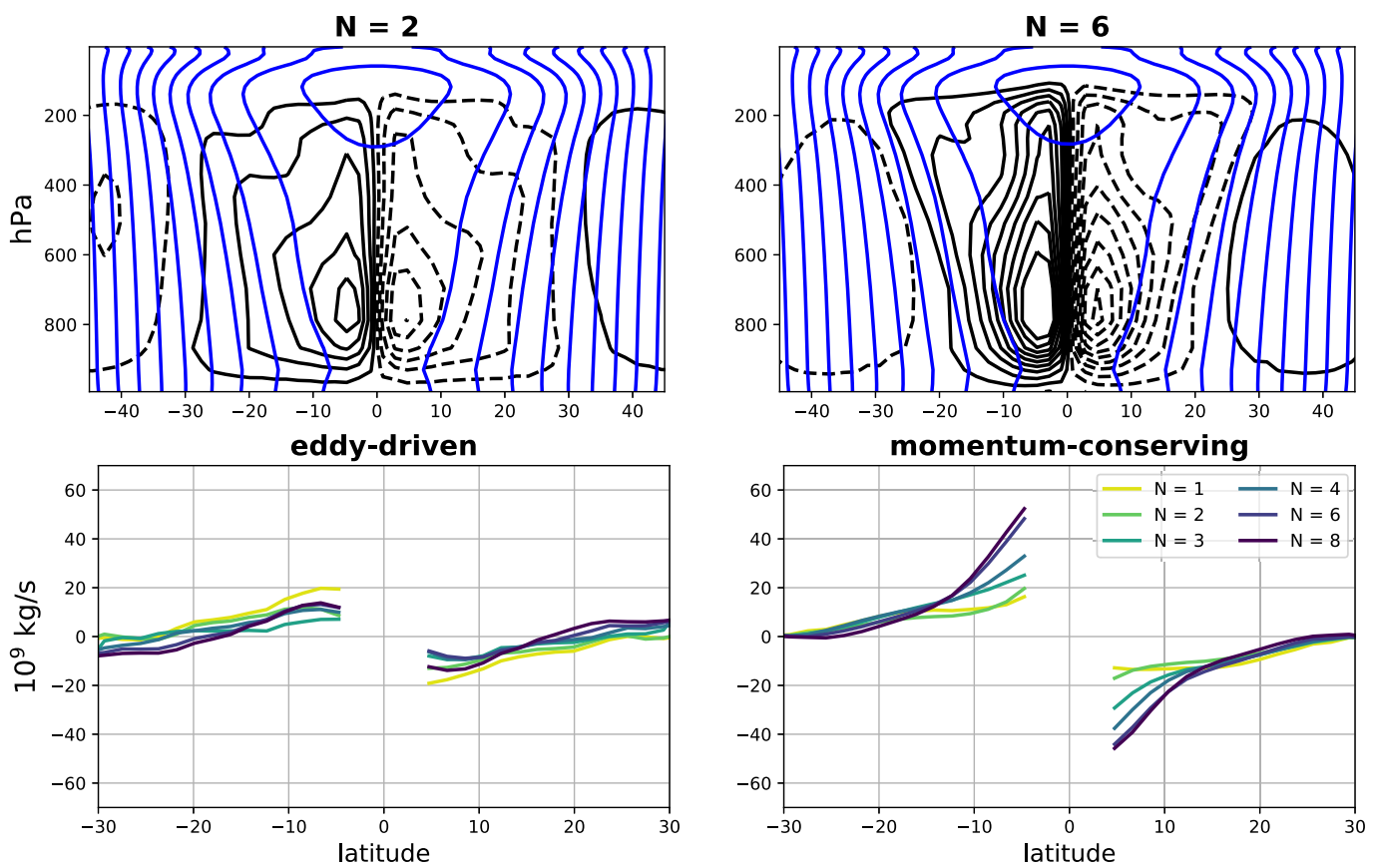

Fig. 8. (top) Anomalous streamfunction (contour interval: $6 \times 10^{9} \mathrm{~kg} \mathrm{~s}^{-1}$ ) for (left) $N=2$ and (right) $N=$ 6 plotted against angular momentum contours for the reference state (contour interval: $1.5 \times$ $10^{8} \mathrm{~kg} \mathrm{~m}^{-2} \mathrm{~s}^{-1}$ ). (bottom) Mass flux component breakdown associated with the (left) eddy-driven and (right) momentum-conserving slowdown mechanisms. Anomalous mass fluxes within $4.2^{\circ}$ of the equator are not shown, as the streamfunction components are poorly defined as the Coriolis parameter approaches zero.

mechanisms driving the slowdown of the HC. We have shown surface warming to be intrinsically tied to the regions of prescribed OHT convergence (Fig. 1c). In the cases of low $N$, localized warming reduces baroclinicity in the midlatitudes, resulting in a weakening of the $\mathrm{HC}$ through eddy momentum fluxes (Walker and Schneider 2006). But, if the OHT is limited to the subtropics (high $N$ ), these momentum fluxes remain relatively unchanged, indicating that the $\mathrm{HC}$ anomaly must be predominantly momentum-conserving in nature, associated with the decrease in the tropical meridional temperature gradient.

To parse out the mechanisms that are driving the slowdown in each case, we consider the steady-state zonal-mean zonal momentum equation assuming small frictional torques above the boundary layer and quasihorizontal flow $(w=0)$ in regions near the latitude of the hemispheric streamfunction extremum:

$$
f\left(1-R_{o}\right)[\bar{v}] \approx S_{e},
$$

where $f$ is the Coriolis parameter, the local Rossby number is $R_{o}=-[\bar{\zeta}] / f, \zeta$ is the relative vorticity, and $S_{e}$ as the horizontal eddy momentum flux divergence. Following Schneider and Bordoni (2008), we can then use $S_{e}$ to approximate the component of the streamfunction associated with eddy momentum fluxes above the boundary layer:

$$
\psi_{e}(\phi, p)=\frac{2 \pi a \cos \phi}{f g} \int_{0}^{p} S_{e} d p^{\prime}
$$

Assuming the difference between $\psi$ and $\psi_{e}$ to be the streamfunction component associated with the mean momentum fluxes, we can use this breakdown to approximate how much of the slowdown in each case can be attributed to its momentum-conserving and eddydriven mechanisms. It is important to note that this approximation is not without its limits. First, because this approximation is limited to above the boundary layer, the streamfunction patterns do not close at the surface. Second, these circulation patterns are poorly defined within $4^{\circ}$ of the equator, where $f$ approaches zero. However, in presenting this breakdown in terms of the mass flux (calculated as the signed maximum of the streamfunction) and neglecting the variations close to the equator, a clear pattern still emerges, shown in the bottom panels of Fig. 8.

In nearly every case, the momentum-conserving component is larger than the eddy-driven component, and the dominance of the momentum-conserving component increases with $N$. Because the warming 
associated with OHT convergence is primarily a local response, subtropical forcing results in a greater decrease in the subtropical meridional SST gradient, weakening the $\mathrm{HC}$ as $N$ increases. On the other hand, this local response limits the effects of the increased OHT on the midlatitude baroclinicity, resulting in minimal decreases in the subtropical upper-level eddy momentum flux divergence. The one exception to this is the $N=1$ case, where $\mathrm{OHT}$ extends to the poles. Amplified warming in the polar regions and midlatitudes [as discussed in Rencurrel and Rose (2018)] reduces midlatitude baroclinicity such that the reduced momentum flux divergence is the majority driver of the slowdown. This works to slightly enhance the HC slowdown when compared to the case of $N=2$, as discussed in section 3. In general, as $N$ increases, the mechanisms driving the slowdown of the $\mathrm{HC}$ become more momentum-conserving in nature, resulting in a streamfunction anomaly tilt that follows the angular momentum contours.

\section{Discussion and conclusions}

In analyzing the $\mathrm{HC}$ response to a wide variety of meridional OHT patterns, we find that not only is the HC's role in atmospheric compensation dependent on the spatial pattern of the prescribed forcing, but that it is also inherently dependent on the dynamical mechanisms driving the change in the overturning circulation. Assuming that the AHT will decrease in response to an increase in OHT out of the tropics (i.e., atmospheric compensation), the energy flux of the $\mathrm{HC}$ will play a role in said decrease. But, if the OHT is limited to the subtropics (high $N$ ), the eddy momentum fluxes from the midlatitudes are relatively unchanged. Thus, the only way for the $\mathrm{HC}$ to reduce its energy transport is through a reduction in the GMS or via a reduction in the momentumconserving part of the HC.

The enhanced reduction in the momentum-conserving $\mathrm{HC}$ results in a change in shape of the streamfunction anomaly as $N$ increases, associated with a tilt in the outer branch of the streamfunction along angular momentum contours. This shape change works to both offset the direct effects of the cell slowdown on the energy flux and enhance the change in GMS, effectively decoupling the energy flux decrease from the change in the circulation rate in spite of a greater slowdown in the HC when compared to the midlatitude forcing patterns $(\operatorname{small} N)$. Overall, this momentum-conserving tilt in the streamfunction anomaly results in a net increase in the energy flux, as the changes in GMS do not fully offset the dynamical effects of the cell deformation.

It is interesting to note that all of this adjustment occurs in conjunction with a fairly robust climate response to an increase in OHT. The HC slowdown affects the climate through cloud changes, quasi-independently of how the compensation in heat transport is achieved, resulting in both a global mean warming and the reduction in the meridional SST gradient in all cases (Herweijer et al. 2005; Burls and Fedorov 2017; Rencurrel and Rose 2018; Hilgenbrink and Hartmann 2018). Increased transport also results in a compensating decrease in the energy flux of the cell in all cases, regardless of the spatial extent of the pattern forced.

Extending our analysis to the full range of OHT amplitudes considered by Rencurrel and Rose (2018), we find the $\mathrm{HC}$ response results to be quite robust, with the exception of very large subtropical forcing (e.g., 3- and 4-PW transport for $N=6$ and 8; not shown). In these cases, the prescribed subtropical sea surface heating is strong enough to destabilize the subsidence regions, effectively creating a doubled ITCZ climate state by driving convection on either side of the equator. This works to shift the overturning circulation poleward, resulting in a slowdown that is primarily eddy-driven in nature as the cells are in a region of greater anomalous upper-level eddy momentum flux divergence, reducing the momentum-conserving deformation associated with subtropical forcing. Watt-Meyer and Frierson (2019) found a similar relationship when using fixed-SST experiments to study the effects of ITCZ narrowing. However we consider these extreme OHT cases to be unrealistic, for reasons discussed below.

We acknowledge that these results are limited by the simplified nature of model setup. First, it is possible that our zonally symmetric aquaplanet configuration overestimates the momentum-conserving nature of the $\mathrm{HC}$ response due to the absence of stationary eddies. Second, while the slab ocean permits an analysis of the climatic impact of a wide range of prescribed OHT patterns, it breaks the dynamical coupling between the $\mathrm{HC}$ and the wind-driven ocean circulation. The mass flux of the lower branch of the $\mathrm{HC}$ and the surface ocean currents are coupled via their Ekman drift associated with the surface stress (Held 2001). This has been shown to have a damping effect on the $\mathrm{HC}$ response to various types of forcing (Schneider et al. 2014; Green and Marshall 2017; Schneider 2017; Kang et al. 2018; Yu and Pritchard 2019). It is likely that the 1-PW OHT anomalies analyzed here are at the upper end of the plausible range of past OHT variations in nature, although we also note that Enderton and Marshall (2009) found tropical OHT changes of order $1 \mathrm{PW}$ in a fully coupled model associated with opening the equatorial passage. Regardless, the main conclusion of this work, namely, that aspects of the energy transport of the $\mathrm{HC}$ are intrinsically linked to the dynamical mechanisms driving the mass transport, is unaffected. 

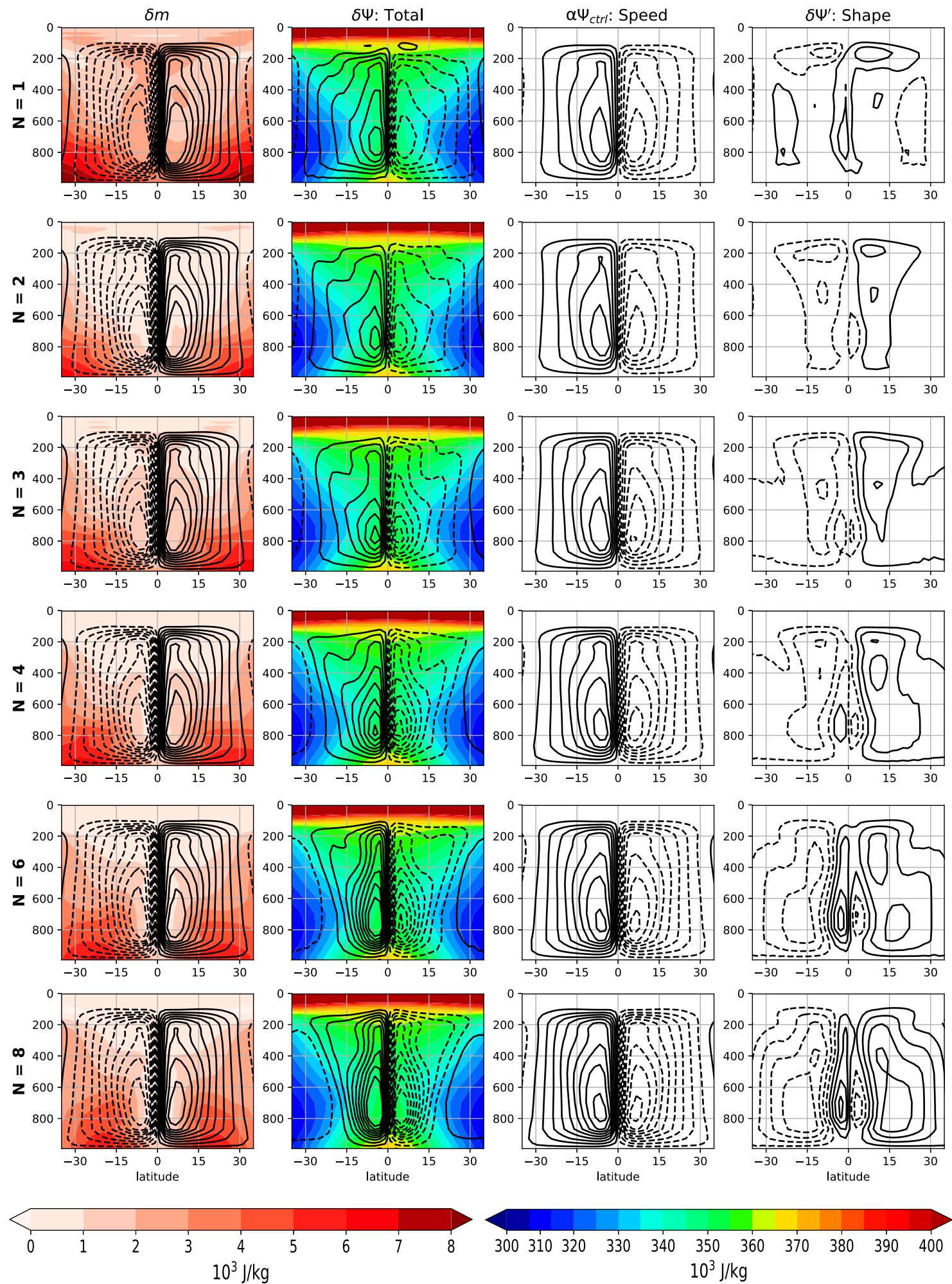

FIG. A1. Anomalous MSE and streamfunction breakdown; as in Fig. 5, but for all $N$. 

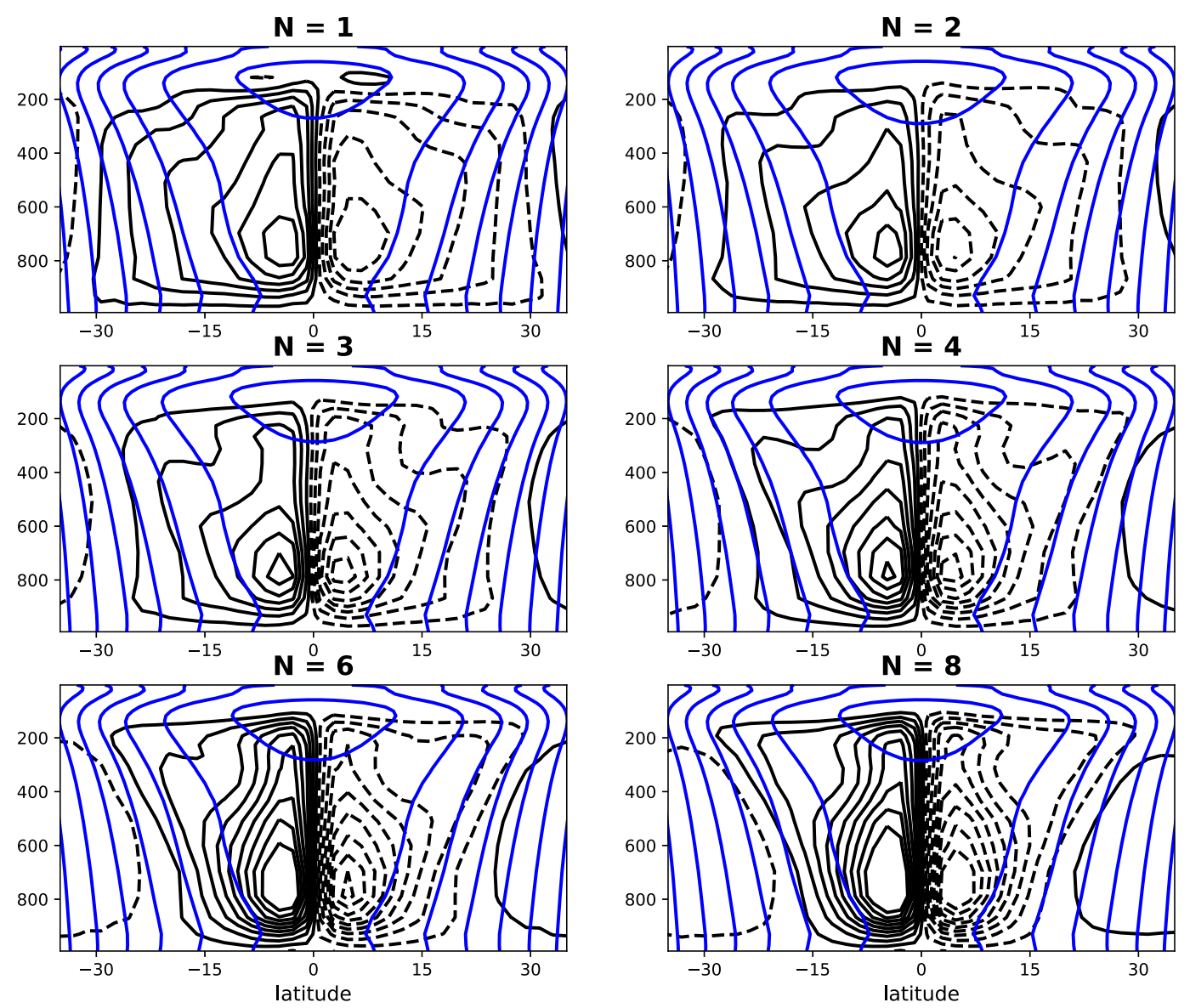

FIG. A2. Anomalous streamfunction plotted against reference angular momentum contours. As in the top panels of Fig. 8, but for all $N$.

Despite the highly idealized nature of these forcing experiments, aspects of the analysis presented here are evident in other recent, more realistic studies. Wei and Bordoni (2018) found that seemingly subtle changes in the vertical profiles of the seasonal $\mathrm{HC}$ and monsoonal circulations can lead to sign changes in the GMS [see also the study by Hill (2019)]. Seo et al. (2017) used a similar breakdown to (7) to study ITCZ shifts response to a doubling of $\mathrm{CO}_{2}$ and found that, as in our work, changes in GMS dominate over the mass flux. In analyzing the hydrological cycles of past warm periods such as the Miocene and Pliocene epochs, Burls and Fedorov (2017) found the HC response to a warming climate to be somewhat muted by the OHT response to paleo-forcings when compared to a quadrupling of $\mathrm{CO}_{2}$, due in part to the reduced tropical meridional temperature gradient.

This work reveals a clear relationship between the HC momentum and energy transport that to the authors' knowledge has not been addressed in the literature previously. A momentum-conserving decrease in the mass flux of the $\mathrm{HC}$, such as that driven by an increase in subtropical OHT, will result in an enhanced decrease in GMS when compared to an eddy-driven mass flux reduction. However, more work is needed to better understand this relationship. Is it possible to predict the deformation of the cell $\left[\psi_{\mathrm{sh}}\right.$ from (9)] directly from the forcing? Advancing our understanding of the relationship between mass and energy transports will play a major role in the development of a prognostic, comprehensive theory for the Hadley cell.

Acknowledgments. We thank Martin Singh, Spencer Hill, and Tim Merlis for their helpful comments and constructive reviews. Complete output from our CESM aquaplanet simulations are publicly available at http:// thredds.atmos.albany.edu:8080/thredds/catalog/cesm/ aquaplanet_som/catalog.html. Individual simulations are labeled with the prefix $Q A q u$ and an indicator of the prescribed OHT, e.g., $Q A q u \_N 2 \_1 P W$. The figure code is publicly available at https:/github.com/mcrencurrel/ The-Efficiency-of-the-Hadley-Cell-Response-to-WideVariations-in-OHT. The CESM project is supported 
primarily by the National Science Foundation. This work was supported by NSF Award AGS-1455071.

\section{APPENDIX}

\section{Full Breakdown of the Circulation and MSE Anomalies for All $N$}

Here we present the full analysis of various aspects of the $\mathrm{HC}$ response to increased OHT. Figure A1 extends the streamfunction breakdown presented in Fig. 5 to all $N$. The maximum increase in MSE is collocated with the region of OHT convergence in all cases, moving equatorward as $N$ increases and reducing the strength of the GMS. Along these same lines, the deformation of the overturning circulation and the associated enhanced slowdown increase with $N$ as well. Although the features discussed in this paper are most prevalent in the cases of subtropical forcing, they are present in all cases when OHT is limited equatorward. This is also true for the anomalous momentum transport of the cell, shown in Fig. A2.

\section{REFERENCES}

Armour, K. C., N. Siler, A. Donohoe, and G. H. Roe, 2019: Meridional atmospheric heat transport constrained by energetic and mediated by large-scale diffusion. J. Climate, 32, 3655-3680, https://doi.org/10.1175/JCLI-D-18-0563.1.

Barreiro, M., A. Cherchi, and S. Masina, 2011: Climate sensitivity to changes in ocean heat transport. J. Climate, 24, 5015-5030, https://doi.org/10.1175/JCLI-D-10-05029.1.

Bice, K. L., C. R. Scotese, D. Seidov, and E. J. Barron, 2000: Quantifying the role of geographic change in Cenozoic ocean heat transport using uncoupled atmosphere and ocean models. Palaeogeogr. Palaeoclimatol. Palaeoecol., 161, 295-310, https:// doi.org/10.1016/S0031-0182(00)00072-9.

Bjerknes, J., 1964: Atlantic air-sea interaction. Advances in Geophysics, 10, 1-82, https://doi.org/10.1016/S00652687(08)60005-9.

Blackburn, M., and B. J. Hoskins, 2013: Context and aims of the aqua-planet experiment. J. Meteor. Soc. Japan, 91A, 1-15, https://doi.org/10.2151/jmsj.2013-A01.

Burls, N. J., and A. V. Fedorov, 2017: Wetter subtropics in a warmer world: Contrasting past and future hydrological cycles. Proc. Natl. Acad. Sci. USA, 114, 12 888-12 893, https:// doi.org/10.1073/pnas.1703421114.

Czaja, A., and J. Marshall, 2006: The partitioning of poleward heat transport between the atmosphere and ocean. J. Atmos. Sci., 63, 1498-1511, https://doi.org/10.1175/JAS3695.1.

Enderton, D., and J. Marshall, 2009: Explorations of atmosphereocean-ice climates on an aquaplanet and their meridional energy transports. J. Atmos. Sci., 66, 1593-1611, https://doi.org/ $10.1175 / 2008$ JAS2680.1.

Fang, M., and K. K. Tung, 1996: A simple model of nonlinear Hadley circulation with an ITCZ: Analytic and numerical solutions. J. Atmos. Sci., 53, 1241-1261, https://doi.org/10.1175/ 1520-0469(1996)053<1241:ASMONH>2.0.CO;2.
Ferreira, D., J. Marshall, and J.-M. Campin, 2010: Localization of deep water formation: Role of atmospheric moisture transport and geometrical constraints on ocean circulation. J. Climate, 23, 1456-1476, https://doi.org/10.1175/2009JCLI3197.1.

,-- , and B. E. J. Rose, 2011: Climate determinism revisited: Multiple equilibria in a complex climate model. J. Climate, 24, 992-1012, https://doi.org/10.1175/2010JCLI3580.1.

Frierson, D. M. W., 2007: The dynamics of idealized convection schemes and their effect on the zonally averaged tropical circulation. J. Atmos. Sci., 64, 1959-1976, https://doi.org/10.1175/ JAS3935.1.

Green, B., and J. Marshall, 2017: Coupling of trade winds with ocean circulation damps ITCZ shifts. J. Climate, 30, 43954411, https://doi.org/10.1175/JCLI-D-16-0818.1.

Held, I. M., 2001: The partitioning of the poleward energy transport between the tropical ocean and atmosphere. J. Atmos. Sci., 58, 943-948, https://doi.org/10.1175/1520-0469(2001)058<0943: TPOTPE $>2.0 . \mathrm{CO} ; 2$.

— , and A. Y. Hou, 1980: Nonlinear axially symmetric circulations in a nearly inviscid atmosphere. J. Atmos. Sci., 37, 515-533, https:// doi.org/10.1175/1520-0469(1980)037<0515:NASCIA >2.0.CO;2.

Herweijer, C., R. Seager, M. Winton, and A. Clement, 2005: Why ocean heat transport warms the global mean climate. Tellus, 57A, 662-675, https://doi.org/10.3402/tellusa.v57i4.14708.

Hilgenbrink, C. C., and D. L. Hartmann, 2018: The response of Hadley circulation extent to an idealized representation of poleward ocean heat transport in an aquaplanet GCM. J. Climate, 31, 9753-9770, https://doi.org/10.1175/JCLI-D-18-0324.1.

Hill, S. A., 2019: Theories for past and future monsoon rainfall changes. Curr. Climate Change Rep., 5, 160-171, https://doi.org/ 10.1007/s40641-019-00137-8.

, Y. Ming, and I. M. Held, 2015: Mechanisms of forced tropical meridional energy flux change. J. Climate, 28, 1725-1742, https://doi.org/10.1175/JCLI-D-14-00165.1.

Hotinski, R. M., and J. R. Toggweiler, 2003: Impact of a Tethyan circumglobal passage on ocean heat transport and "equable" climates. Paleoceanography, 18, 1007, https://doi.org/10.1029/ 2001PA000730.

Kang, S., Y. Shin, and S. Xie, 2018: Extratropical forcing and tropical rainfall distribution: Energetics framework and ocean Ekman advection. npj Climate Atmos. Sci., 1, 20172, https:// doi.org/10.1038/s41612-017-0004-6.

Koll, D. B., and D. S. Abbot, 2013: Why tropical sea surface temperature is insensitive to ocean heat transport changes. J. Climate, 26, 6742-6749, https://doi.org/10.1175/JCLI-D-13-00192.1.

Lee, M.-I., M. J. Suarez, I.-S. Kang, I. M. Held, and D. Kim, 2008: A moist benchmark calculation for atmosphere general circulation models. J. Climate, 21, 4934-4954, https://doi.org/10.1175/ 2008JCLI1891.1.

Liu, Z., H. Yang, C. He, and Y. Zhao, 2016: A theory for Bjerknes compensation: The role of climate feedback. J. Climate, 29, 191-208, https://doi.org/10.1175/JCLI-D-15-0227.1.

Ma, J., R. Chadwick, K.-H. Seo, C. Dong, G. Huang, G. R. Foltz, and J. H. Jiang, 2018: Responses of the tropical atmospheric circulation to climate change and connection to the hydrological cycle. Annu. Rev. Earth Planet. Sci., 46, 549-580, https://doi.org/ 10.1146/annurev-earth-082517-010102.

Merlis, T., T. Schneider, S. Bordoni, and I. Eisenman, 2013: Hadley circulation response to orbital precession. Part I: Aquaplanets. J. Climate, 26, 740-753, https://doi.org/10.1175/ JCLI-D-11-00716.1.

Neale, R. B., J. Richter, S. Park, P. H. Lauritzen, S. J. Vavrus, P. J. Rasch, and M. Zhang, 2013: The mean climate of the 
Community Atmosphere Model (CAM4) in forced SST and fully coupled experiments. J. Climate, 26, 5150-5168, https:// doi.org/10.1175/JCLI-D-12-00236.1.

Neelin, J. D., and I. M. Held, 1987: Modeling tropical convergence based on the moist static energy budget. Mon. Wea. Rev., 115, 3-12, https://doi.org/10.1175/1520-0493(1987) 115<0003:MTCBOT>2.0.CO;2.

Pearson, P. N., and M. Palmer, 2000: Atmospheric carbon dioxide concentrations over the past 60 million years. Nature, 406, 695-699, https://doi.org/10.1038/35021000.

Rencurrel, M. C., and B. E. J. Rose, 2018: Exploring the climatic response to wide variations in ocean heat transport on an aquaplanet. J. Climate, 31, 6299-6318, https://doi.org/10.1175/JCLI-D-17-0856.1.

Rose, B. E. J., 2015: Stable "Waterbelt" climates controlled by tropical ocean heat transport: A nonlinear coupled climate mechanism of relevance to Snowball Earth. J. Geophys. Res. Atmos., 120, 1404-1423, https://doi.org/10.1002/2014JD022659.

— greenhouse in a warm equable climate: A new look at the low gradient paradox. J. Climate, 26, 2117-2136, https://doi.org/ 10.1175/JCLI-D-11-00547.1.

- and M. C. Rencurrel, 2016: The vertical structure of tropospheric water vapor: Comparing radiative and ocean-driven climate changes. J. Climate, 29, 4251-4268, https://doi.org/ 10.1175/JCLI-D-15-0482.1.

—, K. Armour, D. Battisti, N. Feldl, and D. Koll, 2014: The dependence of transient climate sensitivity and radiative feedbacks on the spatial pattern of ocean heat uptake. Geophys. Res. Lett., 41, 1071-1078, https://doi.org/10.1002/2013GL058955.

Satoh, M., 1994: Hadley circulations in radiative-convective equilibrium in an axially symmetric atmosphere. J. Atmos. Sci., 51, 1947-1968, https://doi.org/10.1175/1520-0469(1994)051<1947: HCIREI > 2.0.CO;2.

Schneider, T., 2006: The general circulation of the atmosphere. Annu. Rev. Earth Planet. Sci., 34, 655-688, https://doi.org/ 10.1146/annurev.earth.34.031405.125144.

- 2017: Feedback of atmosphere-ocean coupling on shifts in the intertropical convergence zone. Geophys. Res. Lett., 44, 11 644-11 653, https://doi.org/10.1002/2017GL075817.

, and S. Bordoni, 2008: Eddy-mediated regime transitions in the seasonal cycle of a Hadley circulation and implications for monsoon dynamics. J. Atmos. Sci., 65, 915-934, https://doi.org/ 10.1175/2007JAS2415.1.

— the intertropical convergence zone. Nature, 513, 45-53, https:// doi.org/10.1038/nature13636.

Seo, J., S. M. Kang, and T. M. Merlis, 2017: A model intercomparison of the tropical precipitation response to a $\mathrm{CO}_{2}$ doubling in aquaplanet simulations. Geophys. Res. Lett., 44, 993-1000, https://doi.org/10.1002/2016GL072347.
Singh, M. S., and Z. Kuang, 2016: Exploring the role of eddy momentum fluxes in determining the characteristics of the equinoctial Hadley circulation: Fixed-SST simulations. J. Atmos. Sci., 73, 2427-2444, https://doi.org/10.1175/JAS-D15-0212.1.

,-- , and Y. Tian, 2017: Eddy influences on the strength of the Hadley circulation: Dynamic and thermodynamic perspectives. J. Atmos. Sci., 74, 467-486, https://doi.org/10.1175/ JAS-D-16-0238.1.

Stone, P. H., 1978: Constraints on dynamical transports of energy on a spherical planet. Dyn. Atmos. Oceans, 2, 123-139, https:// doi.org/10.1016/0377-0265(78)90006-4.

Trenberth, K. E., and J. M. Caron, 2001: Estimates of meridional atmosphere and ocean heat transports. Climate Dyn., 14 3433-3443, https://doi.org/10.1175/1520-0442(2001)014<3433: EOMAAO $>2.0 . \mathrm{CO} ; 2$.

- and D. P. Stepaniak, 2003: Covariability of components of poleward atmospheric energy transports on seasonal and interannual timescales. J. Climate, 16,3691-3705, https://doi.org/ 10.1175/1520-0442(2003)016<3691:COCOPA $>2.0 . C O ; 2$.

Vallis, G., P. Zurita-Gotor, C. Cairns, and J. Kidson, 2015: Response of the large-scale structure of the atmosphere to global warming. Quart. J. Roy. Meteor. Soc., 141, 1479-1501, https://doi.org/10.1002/qj.2456.

Voigt, A., and Coauthors, 2016: The tropical rain belts with an annual cycle and continent model intercomparison project: TRACMIP. J. Adv. Model. Earth Syst., 8, 1868-1891, https:// doi.org/10.1002/2016MS000748.

Walker, C. C., and T. Schneider, 2006: Eddy influences on Hadley circulations: Simulations with an idealized GCM. J. Atmos. Sci., 63, 3333-3349, https://doi.org/10.1175/JAS3821.1.

Watt-Meyer, O., and D. M. W. Frierson, 2019: ITCZ width controls on Hadley cell extent and eddy-driven jet position and their response to warming. J. Climate, 32, 1151-1166, https:// doi.org/10.1175/JCLI-D-18-0434.1.

Wei, H.-H., and S. Bordoni, 2018: Energetic constraints on the ITCZ position in idealized simulations with a seasonal cycle. J. Adv. Model. Earth Syst., 10, 1708-1725, https://doi.org/ 10.1029/2018MS001313

Winton, M., 2003: On the climatic impact of ocean circulation. J. Climate, 16, 2875-2889, https://doi.org/10.1175/15200442(2003)016<2875:OTCIOO > 2.0.CO;2.

Wunsch, C., 2005: The total meridional heat flux and its oceanic and atmospheric partition. J. Climate, 18, 4374-4380, https:// doi.org/10.1175/JCLI3539.1.

Yu, S., and M. S. Pritchard, 2019: A strong role for the AMOC in partitioning global energy transport and shifting ITCZ position in response to latitudinally discrete solar forcing in CESM1.2. J. Climate, 32, 2207-2226, https://doi.org/10.1175/ JCLI-D-18-0360.1. 